\title{
Endoscopic submucosal dissection by transnasal endoscope for esophageal cancer with pharyngoesophageal anastomotic stricture after total pharyngo- laryngo-esophagectomy
}

Patients with squamous cell carcinoma (SCC) of the head and neck often develop secondary esophageal carcinomas. We describe a successful case of endoscopic submucosal dissection (ESD) using a transnasal endoscope to treat a patient with esophageal cancer with a pharyngoesophageal anastomotic stricture after total pharyngo-laryngo-esophagectomy (TPLE).

A 60-year-old man was referred to our division for treatment of esophageal cancer. The patient had undergone TPLE for advanced hypopharyngeal cancer 5 years previously, and endoscopy revealed a flat lesion in the lower third of the esophagus ( $\triangleright$ Fig. $\mathbf{1}$ a,b). The patient had a pharyngoesophageal anastomotic stricture after the TPLE that could not be passed using a conventional endoscope ( $\triangleright$ Fig.1 c). Endoscopic balloon dilation was not possible because of the presence of a voice prosthesis ( $\triangleright$ Fig. $1 \mathbf{d}$ ). Recently, an improved transnasal endoscope, the EG-L580NW (Fujifilm, Tokyo, Japan), has become available, with a 5.8-mm outer diameter and a 2.4-mm forceps diameter ( Fig.2a) [1]. An ultrathin
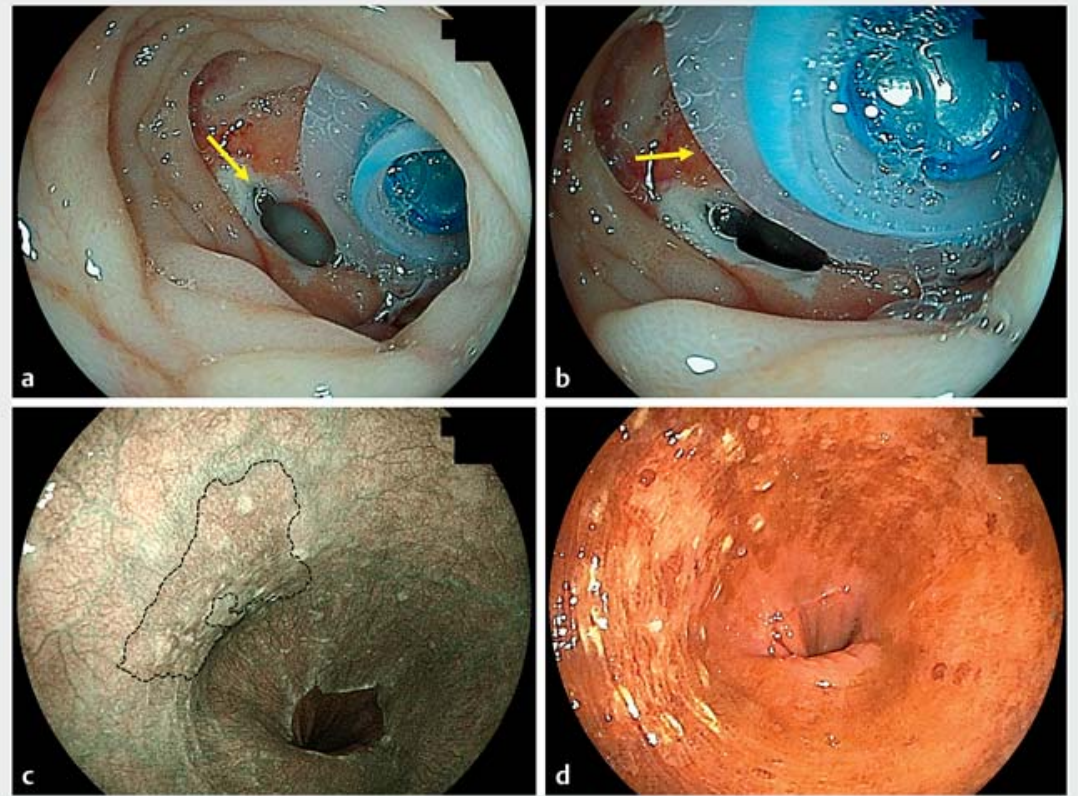

- Fig. 1 a-d A 60-year-old man referred for treatment of esophageal cancer 5 years after undergoing total pharyngo-laryngo-esophagectomy (TPLE) for advanced hypopharyngeal cancer. a Blue laser imaging shows a brownish area in the lower third of the esophagus. b After chromoendoscopy using Lugol's iodine, markers were placed $2-3 \mathrm{~mm}$ outside of the lesion margins. c Pharyngoesophageal anastomotic stricture after the TPLE (arrow). d A voice prosthesis had been used to restore the voice (arrow).
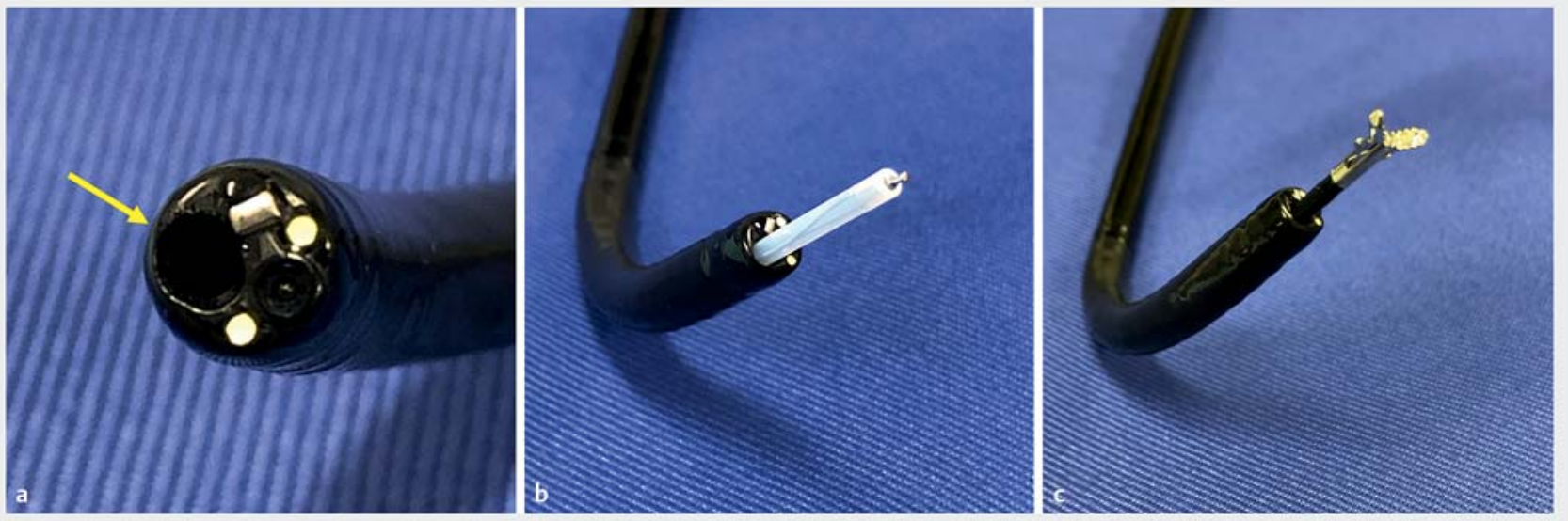

- Fig. 2 a The EG-L580NW (Fujifilm, Tokyo, Japan) has an instrument channel with a $2.4-\mathrm{mm}$ inner diameter. b The Souten ultrathin needletype device has a diameter of $2.35 \mathrm{~mm}$ at the insertion sheath. $\mathbf{c}$ The Raicho hemostasis forceps has a diameter of $2.3 \mathrm{~mm}$ at the insertion sheath. 

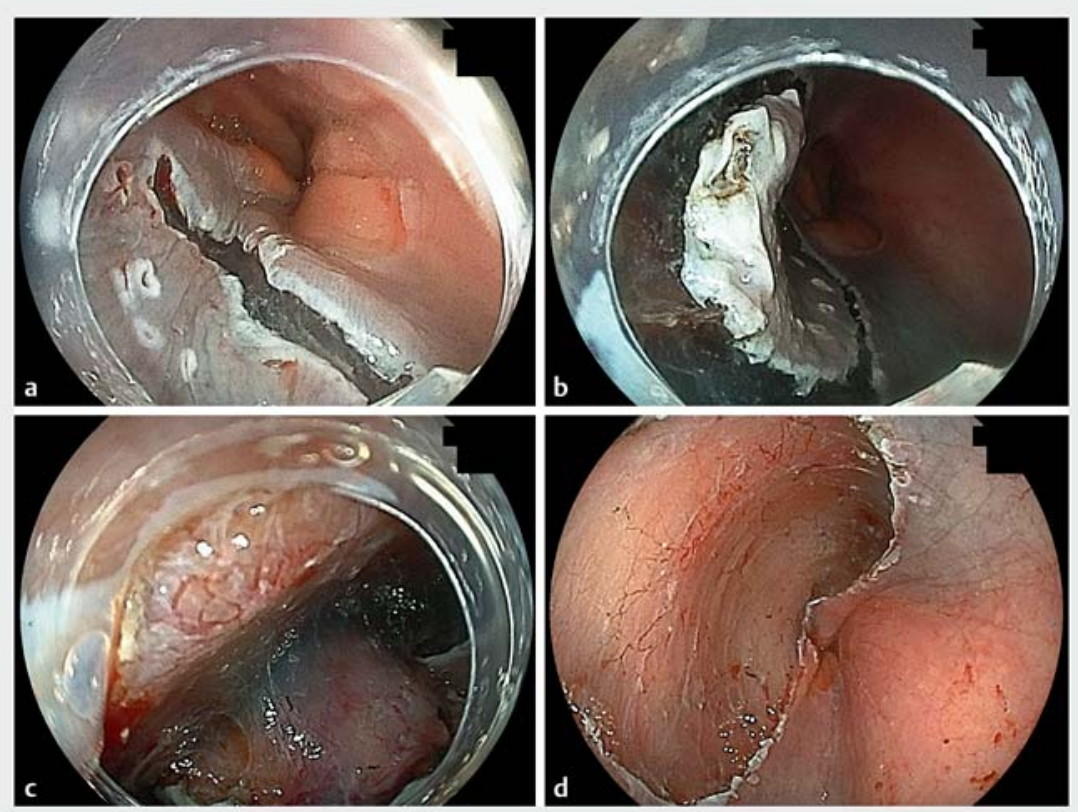

Fig. 3 a A caudal incision was performed. b Circumferential incisions were made using the Souten. c Submucosal dissection was performed. $\mathbf{d}$ En bloc resection was achieved without causing injury to the muscularis propria.
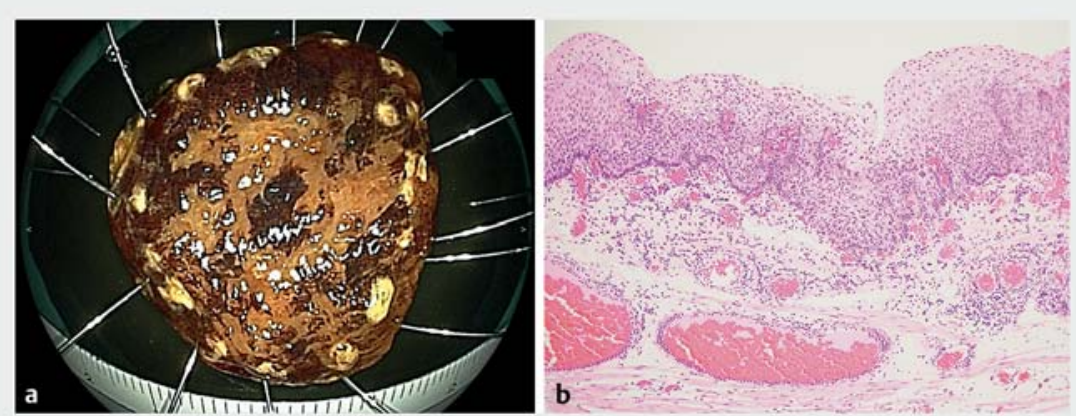

- Fig. 4 a Resected specimen after iodine staining. b Histology shows esophageal squamous cell carcinoma limited to the epithelium. needle-type device (Souten; Kaneka Medics, Tokyo, Japan) and hemostasis forceps (Raicho; Kaneka Medics, Tokyo, Japan) can be used with this endoscope ( Fig. 2b,c) [2]. Thus, we performed ESD using a transnasal endoscope to treat the esophageal cancer ( $\vee$ Video 1 ). Circumferential incisions were made using the Souten with Endo Cut I mode (VIO 300D; Erbe, Tübingen, Germany; effect 2, duration 2, interval 2) ( $\mathbf{F i g . 3}$ a,b). To control bleeding, the Raicho forceps was used in soft coagulation mode at $50 \mathrm{~W}$. Submucosal dissection was performed using the Souten and swift coagulation mode (effect 3, 30 W) ( $\triangleright$ Fig.3c). Finally, en bloc resection was achieved without injury to the mus-

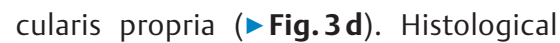
examination revealed $\mathrm{R} 0$ resection of the squamous cell carcinoma, which was limited to the epithelium ( $\mathbf{F i g . 4 ) . ~}$

Esophageal ESD using a transnasal endoscope was useful in treating esophageal cancer with an anastomotic stricture that could not be passed using a conventional endoscope.

Endoscopy_UCTN_Code_TTT_1AO_2AG

Competing interests

The authors declare that they have no conflict of interest.

The authors

Yoshiyasu Kitagawa', Takuto Suzuki ${ }^{1}$, Kentaro Nakamura ${ }^{1,2}$, Rino Nankinzan ${ }^{1}$, Taketo Yamaguchi ${ }^{3}$

1 Endoscopy Division, Chiba Cancer Center, Chiba, Japan

2 Department of Gastroenterology, Toho University Sakura Medical Center, Sakura, Japan

3 Department of Gastroenterology, Chiba Cancer Center, Chiba, Japan 


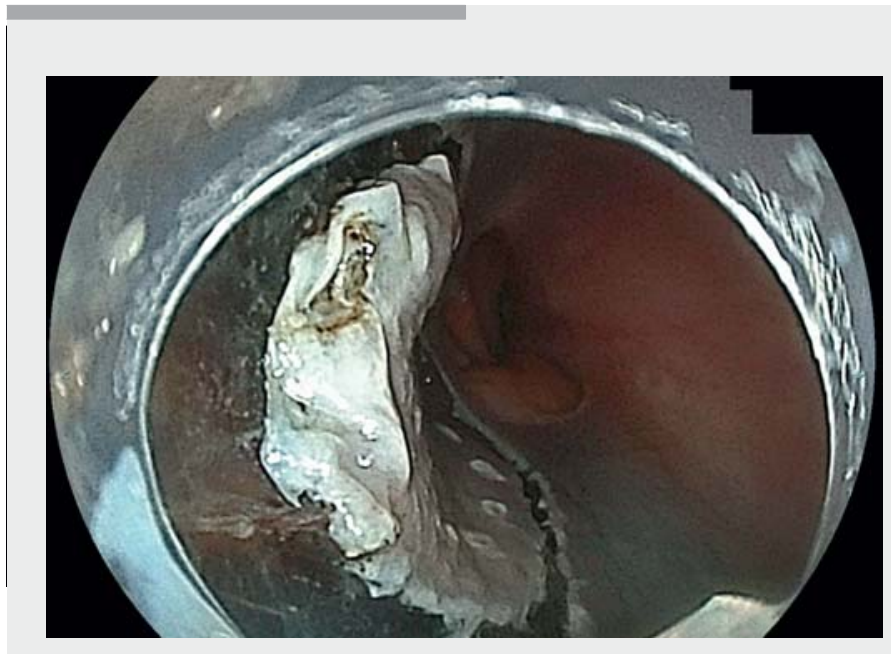

Video 1 Endoscopic submucosal dissection by transnasal endoscope for esophageal cancer with pharyngoesophageal anastomotic stricture after total pharyngo-laryngo-esophagectomy.
Bibliography

Endoscopy 2020; 52: E445-E447

DOI $10.1055 / \mathrm{a}-1158-8948$

ISSN 0013-726X

published online 12.5 .2020

(C) 2020. Thieme. All rights reserved.

Georg Thieme Verlag KG, Rüdigerstraße 14, 70469 Stuttgart, Germany

\section{ENDOSCOPY E-VIDEOS}

https://eref.thieme.de/e-videos

回回 Endoscopy E-Videos is a free 然 回舴: on interesting cases and new techniques in gastroenterological endoscopy. All papers include a high quality video and all contributions are freely accessible online.

This section has its own submission website at

https://mc.manuscriptcentral.com/e-videos

\section{Yoshiyasu Kitagawa, MD, PhD}

Endoscopy Division, Chiba Cancer Center, 666-2 Nitonacho, Chuo-ku, Chiba, Japan Fax: +81-43-2628680

ykitagawa@chiba-cc.jp

\section{References}

[1] Suzuki T, Kitagawa Y, Nankinzan R et al. Early gastric cancer diagnostic ability of ultrathin endoscope loaded with laser light source. World J Gastroenterol 2019; 25: 1378-1386

[2] Minato Y, Sakai E, Ohata K. Conscious transnasal hybrid endoscopic submucosal dissection enables safe and painless en bloc resection in elderly patients with early gastric cancer. VideoGIE 2019; 4: 157-158 